SHORT REPORT

\title{
Effect of massage of the hamstring muscle group on performance of the sit and reach test
}

\author{
A Barlow, R Clarke, N Johnson, B Seabourne, D Thomas, J Gal
}

Br J Sports Med 2004;38:349-351. doi: 10.1136/bjsm.2002.003673

Objective: To investigate if a single massage of the hamstring muscle group would alter the performance of the sit and reach test.

Methods: Before treatment, each of 11 male subjects performed the sit and reach test. The treatment consisted of either massage of the hamstring muscle group (both legs, total time about 15 minutes) or supine rest with no massage. Performance of the sit and reach test was repeated after treatment. Each subject returned the subsequent week to perform the tests again, receiving the alternative treatment relative to their initial visit. Mean percentage changes in sit and reach scores after treatment were calculated for the massage and no massage treatments, and analysed using Student's $t$ tests.

Results: Mean (SD) percentage changes in sit and reach scores after massage and no massage were small $(6.0$ (4.3)\% and $4.6(4.8) \%$ respectively) and not significantly different for subjects with relatively high (15 cm and above) values before treatment. Mean percentage changes in sit and reach scores for subjects with relatively low values before treatment (below $15 \mathrm{~cm}$ ) were large (18.2 (8.2)\% and 15.5 (16.2)\% respectively), but no significant differences were found between the massage and no massage groups.

Conclusions: A single massage of the hamstring muscle group was not associated with any significant increase in sit and reach performance immediately after treatment in physically active young men.

$\mathrm{F}$ lexibility describes the range of motion of a joint. Enhanced flexibility is desirable in locomotor (synovial) joints for a number of reasons. These include increased reach or stride length, and thus, during a repeated activity over a fixed distance/displacement, potentially smaller number of contraction cycles. Activation and deactivation of muscle consumes energy over and above force production, and therefore the fewer times muscle is turned on and off, the less energy is consumed. Equally, greater range of motion effectively means a longer time frame over which muscle contraction can take place. When muscles are allowed sufficient time to accelerate and decelerate limb segments, connective tissues are spared and are therefore less prone to rupture. Thus enhanced flexibility is associated with improved movement economy and reduced risk of injury. ${ }^{1}$

Massage is thought to relax muscle, and could therefore help to enhance joint flexibility by reducing the passive tension of antagonistic muscles. ${ }^{2-4}$ However, experimental results are equivocal. For example, Wiktorsson-Moeller $e t \mathrm{al}^{5}$ found stretching to be the most effective way to increase range of motion in lower limb joints. In contrast, Crosman et $a l^{6}$ found that a single massage of the hamstring muscle group increased the passive range of motion in hip joints.

The purpose of this study was to investigate the effect of a single hamstring massage on the performance of the sit and reach test. This is a popular fitness diagnostic involving the hips, spine, and shoulders. ${ }^{7-9}$ The hip flexion component depends on the strength of the hip flexor agonists and the resistance of the hip flexor antagonists. The hip flexor antagonistic muscles are primarily the members of the hamstring group. Thus a reduction in hamstring passive tension may be expected to result in greater hip flexion and therefore an increased sit and reach score.

\section{METHODS}

\section{Subjects}

This study involved 11 healthy active male volunteers with a mean (SD) age of 21 (3) years, and no history of musculoskeletal disorders. Signed informed consent was obtained from each subject before the start of this ethically approved study.

\section{Procedures}

Subjects were randomly assigned to attend two test sessions each, separated by one week, wherein they would receive either a specific hamstring muscle group massage to both legs by a qualified massage therapist or supine rest. Before and after each treatment session, the remaining investigators would obtain the subjects' sit and reach scores, unaware of the specific treatment details. The sit and reach tests were performed according to the manufacturer's (Cranlea Medical Electronics, Bournville, Birmingham B30 2AH, UK) guidelines (fig 1). In addition, the subjects were blindfolded (reducing psychological influences), and each of the three attempts (best one chosen, as in guidelines) was separated by a 30 second rest in a tucked limb position (minimising connective tissue creep). ${ }^{10}$ The massage treatment (lasting for about 15 minutes) was a specific suite of effleurage and petrissage strokes, delivered to the hamstring muscle group of each leg. Subjects returned the following week to receive the alternative treatment relative to their initial visit, and the sit and reach test procedures were repeated.

\section{Data analysis}

Having obtained the best sit and reach score $(\mathrm{cm})$ per subject, the percentage change from the value before treatment was calculated. Mean percentage changes due to massage or no massage were compared using paired Student's $t$ tests ${ }^{11}$ (SPSS 11.0 for Windows). The null hypothesis was that a single massage treatment to the hamstring muscle group would have no effect on sit and reach performance immediately after treatment. The alternative hypothesis was that a single massage treatment to the hamstring muscle group would result in a change in sit and reach performance after treatment. 


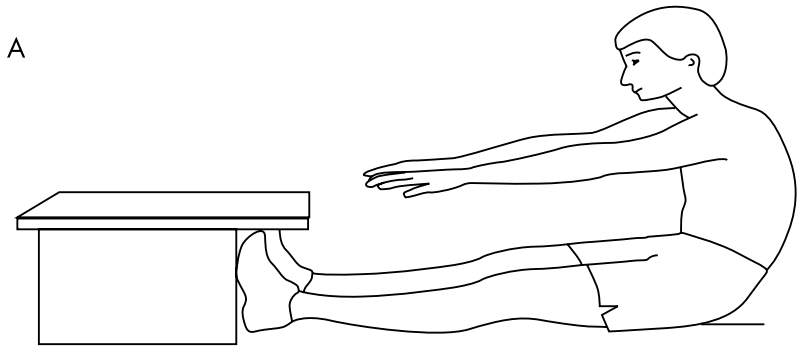

B

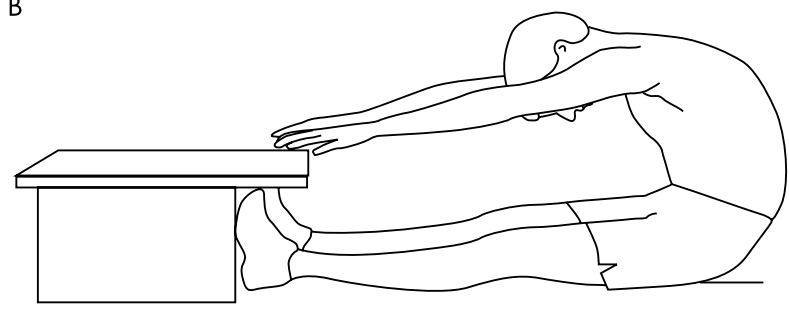

Figure 1 (A) Schematic diagram for the starting position of the sit and reach test. Subjects sit with the soles of their feet against the box, and with their hips flexed to about $90^{\circ}$ to assume an upright sitting position. (B) Schematic diagram of the reaching position of the sit and reach test. Subjects flex the their hip joints and vertebral column (with possible contributions from shoulder joint flexion and scapular elevation) to reach forward as far as possible. A centimetre scale is printed on the top surface of the box. The $15 \mathrm{~cm}$ line is approximately flush with the location of the toes below the overhang of the box.

\section{RESULTS}

Table 1 shows the sit and reach scores $(\mathrm{cm})$ for all 11 subjects for each of their massage or no massage visits. Figures 2 and 3 show the percentage changes in the scores as functions of their respective pretreatment values, for massage and no massage treatments respectively. The $15 \mathrm{~cm}$ toe line boundary is indicated on each figure. Mean (SD) percentage changes in sit and reach scores after massage and no massage were small (6.0 (4.3)\% and $4.6(4.8) \%$ respectively) and not significantly different $(\mathrm{n}=5 ; \mathrm{p}=0.663)$ for subjects with relatively high ( $15 \mathrm{~cm}$ and above) values before treatment. Mean percentage changes in sit and reach scores after massage or no massage for subjects with relatively low reaches (below $15 \mathrm{~cm}$ ) before treatment were considerably larger (18.2 (8.2)\% and $15.5(16.2) \%$ respectively), but not significantly different $(\mathrm{n}=6 ; \mathrm{p}=0.699)$.

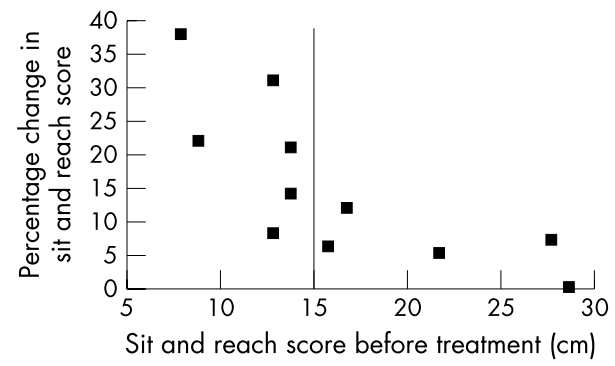

Figure 2 Percentage changes in pretreatment sit and reach scores are plotted against corresponding pretreatment values for the massage treatment. The vertical line indicates the $15 \mathrm{~cm}$ toe line boundary separating the subgroups for statistical comparison.

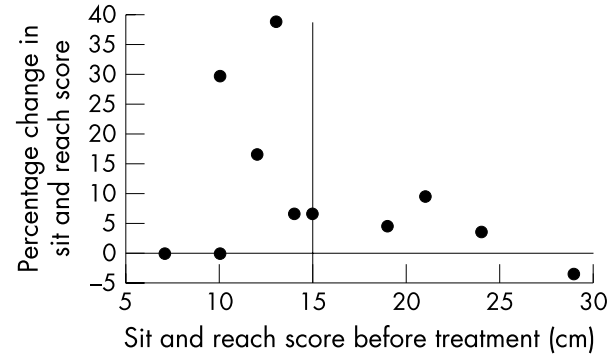

Figure 3 Percentage changes in pretreatment sit and reach scores are plotted against corresponding pretreatment values for the no massage treatment. The vertical line indicates the $15 \mathrm{~cm}$ toe line boundary separating the subgroups for statistical comparison.

\section{DISCUSSION}

The results of this study suggest that a single massage of the hamstring muscle group does not significantly alter sit and reach performance. However, the results also seem to indicate that percentage changes in sit and reach scores may be inversely proportional to pretreatment values. The percentage changes in sit and reach scores were relatively small for those with pretreatment values of $15 \mathrm{~cm}$ and above-that is, those with relatively long reaches to begin with. It has been suggested that the contribution of hip flexion to sit and reach performance is about $60 \%$, with the remainder being derived primarily from spinal column flexion. ${ }^{8}$ If reach was limited by spinal or shoulder flexibility, then hamstring massage would be unlikely to have any effect. This could have been important for the subjects with high reach scores who were perhaps not limited by hamstring muscle resistance in the first place.

For all subjects with relatively low reach scores (below $15 \mathrm{~cm}$ ) before treatment, massage was associated with an increase in reach, supporting the results of Crosman et al. ${ }^{6}$ After the no massage treatment, however, some subjects showed no change, whereas others showed considerable change in their sit and reach ability. Wiktorsson-Moeller et $\mathrm{al}^{5}$ found that massage alone or in combination with a warm up did not significantly increase any lower extremity ranges of motion, except for the ankle joint (dorsiflexion). They found stretching to be the most effective way to improve flexibility in all of the lower extremity joints. ${ }^{5}$ It could be that the subjects with shorter reach who showed an increase in reach after the no massage treatment benefited from a stretching effect of the set of three reaches before treatment. ${ }^{12}$ Perhaps the supine rest itself relaxed the hamstring muscles sufficiently to allow enhanced reach in the subjects with

Table 1 Sit and reach scores $(\mathrm{cm})$ for all subjects for each treatment

\begin{tabular}{cccllll}
\hline & \multicolumn{2}{l}{ Massage } & & \multicolumn{2}{l}{ No massage } & Massage visit 1 \\
\cline { 2 - 3 } Subject & Before & After & & Before & After & or 2 \\
\hline 1 & 17 & 19 & & 19 & 20 & 1 \\
2 & 16 & 17 & & 10 & 13 & 2 \\
3 & 22 & 23 & & 21 & 23 & 1 \\
4 & 9 & 11 & & 15 & 16 & 1 \\
5 & 29 & 29 & 29 & 28 & 2 \\
6 & 14 & 17 & & 14 & 15 & 2 \\
7 & 13 & 17 & 12 & 14 & 2 \\
8 & 28 & 30 & 24 & 25 & 1 \\
9 & 13 & 14 & & 10 & 10 & 2 \\
10 & 14 & 16 & 13 & 18 & 1 \\
11 & 8 & 11 & 7 & 7 & 2 \\
\hline
\end{tabular}




\section{Take home message}

A single massage of the hamstring muscle group seems to have little effect in people who already have good hip joint flexibility. However, those with poor hip joint flexibility may significantly benefit from a one off hamstring massage. Larger subject numbers would be useful in clarifying these results and ideas.

the low sit and reach scores. Larger subject pools would be required to explore these ideas further.

\section{Authors' affiliations}

A Barlow, R Clarke, N Johnson, B Seabourne, D Thomas, J Gal, School of Applied Sciences, University of Glamorgan, Pontypridd, Wales, UK

Correspondence to: Dr Gal, School of Applied Sciences, University of Glamorgan, Trefforest, Pontypridd CF37 1DL, Wales, UK; igal@glam. ac.uk

Accepted 28 April 2003

\section{REFERENCES}

1 Gleim GW, McHugh MP. Flexibility and its effects on sports injury and performance. J Sports Med 1997;24:289-99.

2 Goats GC. Massage: the scientific basis of an ancient art. Part 1. The techniques. Br J Sports Med 1994;28:149-52.

3 Goats GC. Massage: the scientific basis of an ancient art. Part 2. Physiological and therapeutic effects. Br J Sports Med 1994;28:153-6.

4 Hemmings BJ. Physiological, psychological, and performance effects of massage therapy in sport: a review of the literature. Physical Therapy of Sport 2001;2:165-70.

5 Wikłorsson-Moeller M, Oberg B, Ekstrand J, et al. Effect of warming up, massage, and stretching on range of motion and muscle strength in the lower extremity. Am J Sports Med 1983;11:249-52.

6 Crosman L, Chateauvert S, Weisberg J. The effects of massage to the hamstring muscle group on range of motion. J Orthop Sports Phys Ther 1984;6: 168-72.

7 Sallis JF, McKenzie TL, Alcaraz JE. Habitual physical activity and health related physical fitness in fourth-grade children. Am J Dis Child 1993; 147:890-6

8 Sinclair A, Tester $\mathrm{G}$. The sit and reach test: what does it actually measure? Australian Council for Health, Physical Education and Recreation National Journal 1993; Winter:8-13.

9 Hong Y, Li JX, Robinson PD. Balance control, flexibility and cardiorespiratory fitness among older tai chi practitioners. Br J Sports Med 2000;34:29-34.

10 Lakes RS, Vanderby R. Interrelation of creep and relaxation: a modelling approach for ligaments. J Biomech 1999;121:612-15.

11 Mendenhall W. Introduction to probability and statistics. 6th ed. Boston Massachusetts: PWS Publishers, 1983.

12 Rubley MD, Brucker JB, Knight KL, et al. Flexibility retention 3 weeks after a 5-day training regime. Journal of Sport Rehabilitation 2001;10:105-12. 